\title{
The quadratic type of the 2-principal indecomposable modules of the double covers of alternating groups
}

\author{
John Murray \\ Department of Mathematics \& Statistics, National University of Ireland Maynooth, \\ Co Kildare, Ireland
}

\section{A R T I C L E I N F O}

\section{Article history:}

Received 4 April 2018

Available online 17 July 2018

Communicated by Gunter Malle

\section{$M S C$ :}

20C30

$20 \mathrm{C} 20$

Keywords:

Alternating group

Principal indecomposable module

Dual module

Quadratic form

Characteristic 2

Involution

\begin{abstract}
A B S T R A C T
The principal indecomposable modules of the double cover 2. $\mathcal{A}_{n}$ of the alternating group over a field of characteristic 2 are enumerated using the partitions of $n$ into distinct parts. We determine which of these modules afford a non-degenerate 2. $\mathcal{A}_{n}$-invariant quadratic form. Our criterion depends on the alternating sum and the number of odd parts of the corresponding partition.
\end{abstract}

(C) 2018 Elsevier Inc. All rights reserved.

\section{Introduction}

Recall that an element of a finite group $G$ is said to be 2-regular if it has odd order and real if it is conjugate to its inverse. Moreover a real element is strongly real if it is

E-mail address: John.Murray@mu.ie. 
inverted by an involution and otherwise it is said to be weakly real. If $k$ is a field, then a $k G$-module is said to have quadratic type if it affords a non-degenerate $G$-invariant $k$-valued quadratic form. The following is a recent result of R. Gow and the author [3]:

Proposition 1. Suppose that $k$ is an algebraically closed field of characteristic 2. Then for any finite group $G$, the number of isomorphism classes of quadratic type principal indecomposable $k G$-modules is equal to the number of strongly real 2-regular conjugacy classes of $G$.

Our focus here is on the double cover $2 . \mathcal{A}_{n}$ of the alternating group $\mathcal{A}_{n}$. All real 2-regular elements of $\mathcal{A}_{n}$ are strongly real. So every self-dual principal indecomposable $k \mathcal{A}_{n}$-module has quadratic type. On the other hand, 2. $\mathcal{A}_{n}$ may have real 2-regular elements which are not strongly real. In this note we determine which principal indecomposable $k\left(2 . \mathcal{A}_{n}\right)$-modules have quadratic type.

Let $\mathcal{S}_{n}$ be the symmetric group of degree $n$ and let $\mathcal{D}(n)$ be the set of partitions of $n$ which have distinct parts. In $[6,11.5] \mathrm{G}$. James constructed an irreducible $k \mathcal{S}_{n}$-module $D^{\mu}$ for each partition $\mu \in \mathcal{D}(n)$. Moreover, he showed that the $D^{\mu}$ are pairwise nonisomorphic, and every irreducible $k \mathcal{S}_{n^{-}}$-modules is isomorphic to some $D^{\mu}$.

As $\mathcal{A}_{n}$ has index 2 in $\mathcal{S}_{n}$, Clifford theory shows that the restriction $D^{\mu} \downarrow_{\mathcal{A}_{n}}$ is either irreducible or splits into a direct sum of two non-isomorphic irreducible $k \mathcal{A}_{n}$-modules. Moreover, every irreducible $k \mathcal{A}_{n}$-module is a direct summand of some $D^{\mu} \downarrow_{\mathcal{A}_{n}}$.

D. Benson determined [1] which $D^{\mu} \downarrow_{\mathcal{A}_{n}}$ are reducible and we recently determined [8] when the irreducible direct summands of $D^{\mu} \downarrow_{\mathcal{A}_{n}}$ are self-dual (see below for details). Throughout this paper we use $D_{A}^{\mu}$ to denote an irreducible direct summand of $D^{\mu} \downarrow \mathcal{A}_{n}$.

As the centre of $2 . \mathcal{A}_{n}$ acts trivially on any irreducible module, $D_{A}^{\mu}$ can be considered as an irreducible $k\left(2 . \mathcal{A}_{n}\right)$-module, and all irreducible $k\left(2 . \mathcal{A}_{n}\right)$-modules arise in this way.

The alternating sum of a partition $\mu$ is $|\mu|_{a}:=\sum(-1)^{j+1} \mu_{j}$. We use $\ell_{o}(\mu)$ to denote the number of odd parts in $\mu$. So $|\mu|_{a} \equiv \ell_{o}(\mu)(\bmod 2)$ and $|\mu|_{a} \geq \ell_{o}(\mu)$, if $\mu$ has distinct parts. Our result is:

Theorem 2. Let $\mu$ be a partition of $n$ into distinct parts and let $P^{\mu}$ be the projective cover of the simple $k\left(2 . \mathcal{A}_{n}\right)$-module $D_{A}^{\mu}$. Then $P^{\mu}$ has quadratic type if and only if

$$
\frac{n-|\mu|_{a}}{2} \leq 4 m \leq \frac{n-\ell_{o}(\mu)}{2}, \text { for some integer } m
$$

Note that $P^{\mu}$ is a principal indecomposable $k\left(2 . \mathcal{A}_{n}\right)$-module, but is not a $k \mathcal{A}_{n}$-module. Throughout the paper all our modules are left modules. 


\section{Notation}

\subsection{Principal indecomposable modules}

This section consists of statements of well known facts. See [10, Sections 1.1, 1.10, 3.1, 3.6] for details and proofs.

The group algebra of a finite group $G$ over a field $k$ is a $k$-algebra $k G$ together with a distinguished $k$-basis whose elements are identified with the elements of $G$. So each element of $k G$ is unique expressible as $\sum_{g \in G} \lambda_{g} g$, where $\lambda_{g} \in k$ for all $g \in G$. The algebra multiplication in $k G$ is the $k$-linear extension of the group multiplication in $G$.

Multiplication on the left makes $k G$ into a module over itself, the so-called regular $k G$-module. The indecomposable direct summands of $k G$ are called the principal indecomposable $k G$-modules. Each such module has the form $k G e$, where $e$ is a primitive idempotent in $k G$.

Let $P$ be a principal indecomposable $k G$-module. The sum of all simple submodules of $P$ is a simple $k G$-module $S$. Moreover, $P / J(P) \cong S$, where $J(P)$ is the sum of all proper submodules of $P$. So $P$ is the projective cover of $S$. Moreover $P \leftrightarrow S$ establishes a one-to-one correspondence between the isomorphism classes of principal indecomposable $k G$-modules and the isomorphism classes of irreducible $k G$-modules.

Let $(K, R, k)$ be a $p$-modular system for $G$, where $p$ is prime. So $R$ is discrete valuation ring of characteristic 0 , with unique maximal ideal $J$ containing $p$, and $R$ is complete with respect to the topology induced by the valuation. Also $K$ is the field of fractions of $R, k=R / J$ is the residue field of $R$ and $k$ has characteristic $p$. We assume that $K$ and $k$ are splitting fields for all subgroups of $G$.

In this context every principal indecomposable $k G$-module $P$ has a unique lift to a principal indecomposable $R G$-module $\hat{P}$ (this means that $\hat{P}$ is a finitely generated free $R G$-module, which is projective as $R G$-module, and the $k G$-module $\hat{P} / J \hat{P}$ is isomorphic to $P$ ).

A conjugacy class of $G$ is said to be $p$-regular if its elements have order coprime to $p$. The number of isomorphism classes of irreducible $k G$-modules equals the number of $p$-regular conjugacy classes of $G$. So the number of isomorphism classes of principal indecomposable $k G$-modules equals the number of $p$-regular conjugacy classes of $G$.

\subsection{Symplectic and quadratic forms}

A good reference for this section is [5, VII, 8]. A $k G$-module $M$ is said to be self-dual if it is isomorphic to its dual $M^{*}=\operatorname{Hom}_{k}(M, k)$. This occurs if and only if $M$ affords a non-degenerate $G$-invariant $k$-valued bilinear form. A self-dual $M$ has quadratic, orthogonal or symplectic type if it affords a non-degenerate $G$-invariant quadratic form, symmetric bilinear form or symplectic bilinear form, respectively.

If $p \neq 2, \mathrm{R}$. Gow showed that an indecomposable $k G$-module is self-dual if and only if it has orthogonal or symplectic type, and these types are mutually exclusive. See [5, 
VII, 8.11]. W. Willems, and independently J. Thompson [12], showed that the type of a principal indecomposable module coincides with the type of its socle.

If $p=2, \mathrm{P}$. Fong noted that each non-trivial self-dual irreducible $k G$-module has symplectic type. This form is unique up to scalars, by Schur's Lemma. See [5, VII, 8.13]. However now it is possible that the projective cover has neither orthogonal nor symplectic type.

The correspondence $P \leftrightarrow S$ between principal indecomposable $k G$-modules and simple $k G$-modules respects duality. So $P$ is self-dual if and only if $S$ is self-dual. As the number of isomorphism classes of self-dual irreducible $k G$-modules equals the number of real $p$-regular conjugacy classes of $G$, it follows that the number of isomorphism classes of self-dual principal indecomposable $k G$-modules equals the number of real $p$-regular conjugacy classes of $G$.

Recall that $g \mapsto g^{-1}$, for $g \in G$, extends to a $k$-algebra anti-automorphism $x \mapsto x^{o}$ on $k G$ called the contragredient map.

Proposition 3. Let $(K, R, k)$ be a 2-modular system for $G$ and let $\hat{P}$ be a principal indecomposable $R G$-module. Set $P=\hat{P} / J \hat{P}$ and $S=P / \operatorname{rad}(P)$, let $\Phi$ be the character of $\hat{P}$ and let $\varphi$ be the Brauer character of $S$. Then the following are equivalent:

(i) $\hat{P}$ has quadratic type.

(ii) $P$ has quadratic type.

(iii) $P$ has symplectic type.

(iv) There is an involution $t$ in $G$ and a primitive idempotent e in $k G$ such that $P \cong$ $k G e$ and $t^{-1} e t=e^{o}$.

(v) If $B$ is a symplectic form on $S$, then $B(t s, s) \neq 0$, for some involution $t$ in $G$ and some $s$ in $S$.

(vi) $\varphi(g) \notin 2 R$, for some strongly real 2-regular elements $g$ of $G$.

(vii) $\frac{\Phi(g)}{\left|C_{G}(g)\right|} \in 2 R$, for all weakly real 2-regular elements $g$ of $G$.

The equivalence of (i), (ii), (iii) and (iv) was proved in [4] and that of (ii), (vi) and (vii) in [3]. We only need the equivalence of (ii) and (v) to prove Theorem 2. This was first demonstrated in [7].

\section{The double covers of alternating groups}

\subsection{Strongly real classes}

The alternating group $\mathcal{A}_{n}$ is the subgroup of even permutations in the symmetric group $\mathcal{S}_{n}$. So $\mathcal{A}_{5}, \mathcal{A}_{6}, \ldots$ is an infinite family of finite simple groups. For $n \geq 4, \mathcal{A}_{n}$ has a unique double cover 2. $\mathcal{A}_{n}$. Then 2. $\mathcal{A}_{n}$ is a subgroup of each double cover $2 . \mathcal{S}_{n}$ of $\mathcal{S}_{n}$. Moreover 2. $\mathcal{A}_{n}$ is a Schur covering group of $\mathcal{A}_{n}$, if $n=5$ or $n \geq 8$. In this section we 
describe the conjugacy classes and characters of these groups. See [11] for an elegant exposition of this theory.

Given distinct $i_{1}, i_{2}, \ldots, i_{m} \in\{1, \ldots, n\}$, we use $\left(i_{1}, i_{2}, \ldots, i_{m}\right)$ to denote an $m$-cycle in $\mathcal{S}_{n}$. So $\left(i_{1}, i_{2}, \ldots, i_{m}\right)$ maps $i_{j}$ to $i_{j+1}$, for $j=1, \ldots, m-1$, sends $i_{m}$ to $i_{1}$ and fixes all $i \neq i_{1}, \ldots, i_{m}$. Now each permutation $\sigma \in \mathcal{S}_{n}$ has a unique factorization as a product of disjoint cycles. If we arrange the lengths of these cycles in a non-increasing sequence, we get a partition of $n$, which is called the cycle type of $\sigma$. The set of permutations with a fixed cycle type $\lambda$ is a conjugacy class of $\mathcal{S}_{n}$, here denoted $C_{\lambda}$. In particular the 2-regular conjugacy classes of $\mathcal{S}_{n}$ are indexed by the set $\mathcal{O}(n)$ of partitions of $n$ whose parts are odd.

A transposition in $\mathcal{S}_{n}$ is a 2-cycle $(i, j)$ where $i, j$ are distinct elements of $\{1, \ldots, n\}$. So $(i, j)$ has cycle type $\left(2,1^{n-2}\right)$. It is clear that there is one conjugacy class of involutions for each partition $\left(2^{m}, 1^{n-2 m}\right)$ of $n$, with $1 \leq m \leq n / 2$. We call a product of $m$-disjoint transpositions an $m$-involution in $\mathcal{S}_{n}$. It follows that $\mathcal{S}_{n}$ has $\left\lfloor\frac{n}{2}\right\rfloor$ conjugacy classes of involutions; the $m$-involutions, for $1 \leq m \leq n / 2$.

Suppose that $\pi=\left(i_{1}, i_{1+m}\right)\left(i_{2} i_{2+m}\right) \ldots\left(i_{m}, i_{2 m}\right)$ is an $m$-involution in $\mathcal{S}_{n}$. Then we say that $\left(i_{1}, i_{1+m}\right),\left(i_{2} i_{2+m}\right), \ldots,\left(i_{m}, i_{2 m}\right)$ are the transpositions in $\pi$ and write $\left(i_{j}, i_{j+m}\right) \in \pi$, for $j=1, \ldots, m$. Notice that each $\left\{i_{j}, i_{j+m}\right\}$ is a non-singleton orbit of $\pi$ on $\{1, \ldots, n\}$.

Let $\lambda$ be a partition of $n$. We use $\ell(\lambda)$ to denote the number of parts in $\lambda$, and we say that $\lambda$ is even if $n \equiv \ell(\lambda) \bmod 2$. Then $C_{\lambda} \subseteq \mathcal{A}_{n}$ if and only if $\lambda$ is even, and if $\lambda$ is even, then $C_{\lambda}$ is a union of two conjugacy classes of $\mathcal{A}_{n}$ if $\lambda$ has distinct odd parts and otherwise $C_{\lambda}$ is a single conjugacy class of $\mathcal{A}_{n}$. In either case we use $C_{\lambda, A}$ to denote an $\mathcal{A}_{n}$-conjugacy class contained in $C_{\lambda}$. If $\lambda$ has distinct odd parts then $C_{\lambda, A}$ is a real conjugacy class of $\mathcal{A}_{n}$ if and only if $n \equiv \ell(\lambda) \bmod 4$.

Next let $z \in 2 . \mathcal{A}_{n}$ be the involution which generates the centre of $2 . \mathcal{A}_{n}$. As $\langle z\rangle$ is a central 2-subgroup of 2. $\mathcal{A}_{n}$, there is a one-to-one correspondence between the 2-regular conjugacy classes of $2 . \mathcal{A}_{n}$ and the 2-regular conjugacy classes of $\mathcal{A}_{n} \cong\left(2 . \mathcal{A}_{n}\right) /\langle z\rangle$; if $\lambda$ is an odd partition of $n$ the preimage of $C_{\lambda, A}$ in 2. $\mathcal{A}_{n}$ consists of a single class $\hat{C}_{\lambda, A}$ of odd order elements and another class $z \hat{C}_{\lambda, A}$ of elements whose 2-parts equal $z$.

Notice that an $m$-involution belongs to $\mathcal{A}_{n}$ if and only if $m$ is even. Moreover, the $2 m$-involutions form a single conjugacy class of $\mathcal{A}_{n}$. So $\mathcal{A}_{n}$ has $\left\lfloor\frac{n}{4}\right\rfloor$ conjugacy classes of involutions; the $2 m$-involutions, for $1 \leq m \leq n / 4$. Now each $2 m$-involution in $\mathcal{A}_{n}$ is the image of two involutions in 2. $\mathcal{A}_{n}$, if $m$ is even, or is the image of two elements of order 4 in $2 . \mathcal{A}_{n}$, if $m$ is odd.

Set $m_{o}(\lambda)$ as the number of parts which occur with odd multiplicity in $\lambda$.

Lemma 4. If $\lambda$ is a partition of $n$ with all parts odd then $\hat{C}_{\lambda, A}$ is a strongly real conjugacy class of $2 . \mathcal{A}_{n}$ if and only if there is an integer $m$ such that $\frac{n-\ell(\lambda)}{2} \leq 4 m \leq \frac{n-m_{o}(\lambda)}{2}$. 
Proof. Let $\sigma \in \mathcal{A}_{n}$ have cycle type $\lambda$ and let $\pi$ be an $m$-involution in $\mathcal{S}_{n}$ which inverts $\sigma$. Set $\ell:=\ell(\lambda)$, and let $X_{1}, \ldots, X_{\ell}$ be the orbits of $\sigma$ on $\{1, \ldots, n\}$. Then $\pi$ permutes the sets $X_{1}, \ldots, X_{\ell}$.

If $\pi X_{j}=X_{j}$, for some $j$, then $\pi$ fixes a unique element of $X_{j}$, and hence acts as an $\frac{\left|X_{j}\right|-1}{2}$-involution on $X_{j}$. If instead $\pi X_{j} \neq X_{j}$, then $\pi$ is a bijection $X_{j} \rightarrow \pi X_{j}$. So $\pi$ acts as an $\left|X_{j}\right|$-involution on $X_{j} \cup \pi X_{j}$. We may order the $X_{j}$ and choose $k \geq 0$ such that $\pi X_{j}=X_{j+k}$, for $j=1, \ldots, k$, and $\pi X_{j}=X_{j}$, for $j=2 k+1,2 k+2, \ldots, \ell$. Then from above

$$
m=\sum_{j=1}^{k} \frac{\left|X_{j}\right|+\left|X_{j+k}\right|}{2}+\sum_{j=2 k+1}^{\ell} \frac{\left|X_{j}\right|-1}{2}=\frac{n+2 k-\ell}{2} .
$$

Now the maximum value of $2 k$ is $2 k=\ell-m_{o}(\lambda)$, when $\pi$ pairs the maximum number of orbit of $\sigma$ which have equal size. This implies that $m \leq \frac{n-m_{o}(\lambda)}{2}$. The minimum value of $2 k$ is 0 . This occurs when $\pi$ fixes each orbit of $\sigma$. It follows from this that $m \geq \frac{n-\ell(\lambda)}{2}$.

Conversely, it is clear that for each $m>0$ with $\frac{n-\ell}{2} \leq m \leq \frac{n-m_{o}(\lambda)}{2}$, there is an $m$-involution $\pi \in \mathcal{S}_{n}$ which inverts $\sigma ; \pi$ pairs $\ell+2 m-n$ orbits of $\sigma$ and fixes the remaining $n-2 m$ orbits of $\sigma$. The conclusion of the Lemma now follows from our description of the involutions in $2 \cdot \mathcal{A}_{n}$.

\subsection{Irreducible modules}

By an $n$-tabloid we mean an indexed collection $R=\left(R_{1}, \ldots R_{\ell}\right)$ of non-empty subsets of $\{1, \ldots, n\}$ which are pairwise disjoint and whose union is $\{1, \ldots, n\}$ (also known as an ordered partition of $\{1, \ldots, n\})$. We shall refer to $R_{1}, \ldots, R_{\ell}$ as the rows of $R$. Set $\lambda_{i}:=\left|R_{i}\right|$. Then we may choose indexing so that $\lambda=\left(\lambda_{1}, \ldots, \lambda_{\ell}\right)$ is a partition of $n$, which we call the type of $R$. Now $\mathcal{S}_{n}$ acts on all $\lambda$-tabloids; the corresponding permutation module (over $\mathbb{Z}$ ) is denoted $M^{\lambda}$.

Next recall that the Young diagram of $\lambda$ is a collection of boxes in the plane, oriented in the Anglo-American tradition: the first row consists of $\lambda_{1}$ boxes. Then for $i=2, \ldots, \ell$ in turn, the $i$-th row consists of $\lambda_{i}$ boxes placed directly below the $(i-1)$-th row, with the leftmost box in row $i$ directly below the leftmost box in row $i-1$.

By a $\lambda$-tableau we shall mean a bijection $t:[\lambda] \rightarrow\{1, \ldots, n\}$, or a filling of the boxes in the Young diagram with the symbols $1, \ldots, n$. So for $1 \leq r \leq \ell$ and $1 \leq c \leq \lambda_{r}$, we use $t(r, c)$ to denote the image of the position $(r, c) \in[\lambda]$ in $\{1, \ldots, n\}$. Conversely, given $i \in\{1, \ldots, n\}$, there is a unique $r=r_{t}(i)$ and $c=c_{t}(i)$ such that $t(r, c)=i$. We say that $i$ is in row $r$ and column $c$ of $t$.

Clearly there are $n$ ! tableaux of type $\lambda$ and $\mathcal{S}_{n}$ acts regularly on the set of $\lambda$-tableau. For $\sigma \in \mathcal{S}_{n}$, we define $\sigma t:[\lambda] \rightarrow\{1, \ldots, n\}$ as the composition $(\sigma t)(r, c)=\sigma(t(r, c))$, for all $(r, c) \in[\lambda]$. In other words, the permutation module of $\mathcal{S}_{n}$ acting on tableau is (non-canonically) isomorphic to the regular module $\mathbb{Z} \mathcal{S}_{n}$; once we fix $t$, we may identify $\sigma \in \mathcal{S}_{n}$ with the tableau $\sigma t$. 
Associated with $t$, we have two important subgroups of $\mathcal{S}_{n}$. The column stabilizer of $t$ is $C_{t}:=\left\{\sigma \in \mathcal{S}_{n} \mid c_{t}(i)=c_{t}(\sigma i)\right.$, for $\left.i=1, \ldots, n\right\}$ and the row stabilizer of $t$ is $R_{t}:=\left\{\sigma \in \mathcal{S}_{n} \mid r_{t}(i)=r_{t}(\sigma i)\right.$, for $i=1, \ldots, n$.

We use $\{t\}$ to denote the tabloid formed by the rows of $t$. So $\{t\}_{r}:=\{t(r, c) \mid 1 \leq c \leq$ $\left.\lambda_{r}\right\}$, for $r=1, \ldots, \ell$. Also $\{s\}=\{t\}$ if and only if $s=\sigma t$, for some $\sigma \in R_{t}$. Notice that the actions of $\mathcal{S}_{n}$ on tableau and tabloids are compatible, in the sense that $\sigma\{t\}=\{\sigma t\}$. In other words, the map $t \mapsto\{t\}$ induces a surjective $\mathcal{S}_{n}$-homomorphism $\mathbb{Z} \mathcal{S}_{n} \rightarrow M^{\lambda}$. The kernel of this homomorphism is the $\mathbb{Z}$-span of $\left\{\sigma t \mid \sigma \in R_{t}\right\}$.

The polytabloid $e_{t}$ associated with $t$ is the following element of $M^{\lambda}$ :

$$
e_{t}:=\sum_{\sigma \in C_{t}} \operatorname{sgn}(\sigma)\{\sigma t\}
$$

We use $\operatorname{supp}(t):=\left\{\{\sigma t\} \mid \sigma \in C_{t}\right\}$ to denote the set of tabloids which occur in the definition of $e_{t}$. Note that $e_{\pi t}=\operatorname{sgn}(\pi) e_{t}$, for all $\pi \in C_{t}$. In particular, if $r_{t}(i)=r_{t}(j)$, then $e_{(i, j) t}=-e_{t}$. Also if $\pi \in \mathcal{S}_{n}$, then $C_{\pi t}=\pi C_{t} \pi^{-1}$ and $R_{\pi t}=\pi R_{t} \pi^{-1}$. So $e_{\pi t}=\pi e_{t}$ and $\operatorname{supp}(\pi t)=\pi \operatorname{supp}(t)$.

The $\mathbb{Z}$-span of all $\lambda$-polytabloids is a $\mathcal{S}_{n}$-submodule of $M^{\lambda}$ called the Specht module.

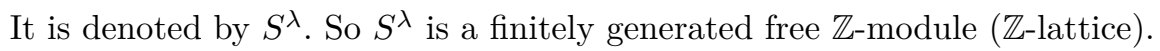

\subsection{Involutions and bilinear forms}

Let $\mu$ be a partition of $n$ which has distinct parts and let $\langle$,$\rangle be the symmetric$ bilinear form on $M^{\mu}$ with respect to which the $\mu$-tabloids form an orthonormal basis. Now let $k$ be a field of characteristic 2. Then according to James, $D^{\mu}:=S^{\mu} / S^{\mu} \cap\left(S^{\mu}\right)^{\perp}$ is a non-zero irreducible $k \mathcal{S}_{n}$-module. Here $\left(S^{\mu}\right)^{\perp}:=\left\{m \in M^{\mu} \mid\langle m, s\rangle \in 2 \mathbb{Z}, \forall s \in S^{\mu}\right\}$.

Suppose that $\mu$ has parts $\mu_{1}>\cdots>\mu_{2 s-1}>\mu_{2 s} \geq 0$. Benson's classification of the irreducible $k \mathcal{A}_{n}$-modules [1], and our classification of the self-dual irreducible $k \mathcal{A}_{n}$-modules [8], are given by:

Lemma 5. $D^{\mu} \downarrow_{\mathcal{A}_{n}}$ is reducible if and only if for each $j>0$

$$
\text { (i) } \mu_{2 j-1}-\mu_{2 j}=1 \text { or } 2 \quad \text { and }\left(\text { ii) } \mu_{2 j-1}+\mu_{2 j} \not \equiv 2(\bmod 4)\right. \text {. }
$$

If $D^{\mu} \downarrow_{\mathcal{A}_{n}}$ is reducible, its irreducible direct summands are self-dual if and only if $\sum_{j>0} \mu_{2 j}$ is even.

Let $\bar{n}$ denote the residue of an integer $n \bmod 2$. Then

Lemma 6. Let $\phi: S^{\mu} \rightarrow D^{\mu}$ be the $\mathbb{Z} \mathcal{S}_{n}$-projection. Then $B(\phi x, \phi y):=\overline{\langle x, y\rangle}$, for $x, y \in$ $S^{\mu}$, defines a non-zero symplectic bilinear form on $D^{\mu}$, if $\mu \neq(n)$. 
Remark 7. Notice that if $x, y \in D^{\mu}$ and $\pi$ is an involution in $\mathcal{S}_{n}$ then

$$
B(\pi(x+y), x+y)=B(\pi x, x)+B(\pi y, y)
$$

So we can focus on a single polytabloid in $S^{\lambda}$.

Lemma 8. If $t$ is a $\mu$-tableau and $\pi$ is an involution in $\mathcal{S}_{n}$, then

$$
\left\langle\pi e_{t}, e_{t}\right\rangle \equiv|\{T \in \operatorname{supp}(\pi t) \cap \operatorname{supp}(t) \mid \pi T=T\}| \bmod 2 .
$$

In particular, if $\left\langle\pi e_{t}, e_{t}\right\rangle$ is odd, then $\pi \in R_{\sigma t}$, for some $\sigma \in C_{t}$.

Proof. We have

$$
\begin{aligned}
\left\langle\pi e_{t}, e_{t}\right\rangle & =\sum_{\sigma_{1}, \sigma_{2} \in C_{t}} \operatorname{sgn}\left(\pi \sigma_{1} \pi^{-1}\right) \operatorname{sgn}\left(\sigma_{2}\right)\left\langle\left(\pi \sigma_{1}\{t\}, \sigma_{2}\{t\}\right\rangle\right. \\
& \equiv\left|\left\{\left(\sigma_{1}, \sigma_{2}\right) \in C_{t} \times C_{t} \mid \pi \sigma_{1}\{t\}=\sigma_{2}\{t\}\right\}\right|(\bmod 2) \\
& =|\operatorname{supp}(\pi t) \cap \operatorname{supp}(t)| .
\end{aligned}
$$

Now notice that $T \mapsto \pi T$ is an involution on $\operatorname{supp}(\pi t) \cap \operatorname{supp}(t)$. So $|\operatorname{supp}(\pi t) \cap \operatorname{supp}(t)| \equiv$ $|\{T \in \operatorname{supp}(\pi t) \cap \operatorname{supp}(t) \mid \pi T=T\}| \bmod 2$.

Suppose that $\left\langle\pi e_{t}, e_{t}\right\rangle$ is odd. Then by the above, there exists $\sigma \in C_{t}$ such that $\pi\{\sigma t\}=\{\sigma t\}$. This means that $\pi \in R_{\sigma t}$.

Lemma 9. Let $t$ be a $\mu$-tableau and let $m$ be a positive integer such that $\left\langle\pi e_{t}, e_{t}\right\rangle$ is odd, for some $m$-involution $\pi \in \mathcal{S}_{n}$. Then $m \leq \frac{n-\ell_{o}(\mu)}{2}$ and $\pi$ fixes at most one entry in each column of $t$.

Proof. By the previous Lemma, we may assume that $\pi \in R_{t}$. Now $R_{t} \cong \mathcal{S}_{\mu}$. For $i>0$, there is $j$-involution in $\mathcal{S}_{i}$ for $j=1, \ldots\left\lfloor\frac{i}{2}\right\rfloor$. So there is an $m$-involution in $R_{t}$ if and only if

$$
m \leq \sum\left\lfloor\frac{\mu_{i}}{2}\right\rfloor=\sum_{\mu_{i} \text { even }} \frac{\mu_{i}}{2}+\sum_{\mu_{i} \text { odd }} \frac{\mu_{i}-1}{2}=\frac{n-\ell_{o}(\mu)}{2}
$$

Let $i, j$ belong to a single column of $t$. We claim that $i, j$ belong to different columns of $\pi t$. For suppose otherwise. Then $(i, j) \in C_{t} \cap C_{\pi t}$. So the map $T \mapsto(i, j) T$ is an involution on $\operatorname{supp}(\pi t) \cap \operatorname{supp}(t)$ which has no fixed-points. In particular $|\operatorname{supp}(\pi t) \cap \operatorname{supp}(t)|$ is even, contrary to hypothesis. This proves the last assertion.

We can now prove a key technical result:

Lemma 10. Let $t$ be a $\mu$-tableau and let $m$ be a positive integer such that $\left\langle\pi e_{t}, e_{t}\right\rangle$ is odd, for some $m$-involution $\pi \in \mathcal{S}_{n}$. Then $m \geq \frac{n-|\mu|_{a}}{2}$. 
Proof. Let $T \in \operatorname{supp}(\pi t) \cap \operatorname{supp}(t)$ such that $\pi T=T$. Write $\pi_{j}$ for the restriction of $\pi$ to the rows $T_{2 j-1}$ and $T_{2 j}$ of $T$, for each $j>0$. Then there is $m_{j} \geq 0$ such that $\pi_{j}$ is an $m_{j}$-involution, for each $j>0$. So $m=\sum m_{j}$ and $\pi=\pi_{1} \pi_{2} \ldots \pi_{\left\lfloor\frac{\ell(\mu)+1}{2}\right\rfloor}$.

We assume for the sake of contradiction that $m<\frac{n-|\mu|_{a}}{2}$. Now $\frac{n-|\mu|_{a}}{2}=\sum_{j>0} \mu_{2 j}$. So $m_{j}<\mu_{2 j}$ for some $j>0$, and we choose $j$ to be the smallest such positive integer.

There is a unique $\sigma \in C_{t}$ such that $T=\{\sigma t\}$. Set $s=\sigma t$. So $\pi \in R_{s}$. We define the graph $\operatorname{Gr}_{\pi}(s)$ of $\pi$ on $s$ as follows. The vertices of $\operatorname{Gr}_{\pi}(s)$ are labels $1, \ldots, \mu_{2 j-1}$ of the columns which meet row $\mu_{2 j-1}$ of $s$. There is an edge $c_{1} \longleftrightarrow c_{2}$ if and only if one of the two transpositions $\left(s\left(2 j-1, c_{1}\right), s\left(2 j-1, c_{2}\right)\right)$ or $\left(s\left(2 j, c_{1}\right), s\left(2 j, c_{2}\right)\right)$ belongs to $\pi_{j}$. As there are at most two entries in each column of $s$ which are moved by $\pi_{j}$, it follows that each connected component of $\operatorname{Gr}_{\pi}(s)$ is either a line segment or a simple closed curve.

We claim that $\operatorname{Gr}_{\pi}(s)$ has a component with a vertex set contained in $\left\{1, \ldots, \mu_{2 j}\right\}$. For otherwise every component $\Gamma$ of $\operatorname{Gr}_{\pi}(s)$ is a line segment and $|\operatorname{Edge}(\Gamma)| \geq \mid \operatorname{Vx}(\Gamma) \cap$ $\left\{1, \ldots, \mu_{2 j}\right\} \mid$. Summing over all $\Gamma$ we get the contradiction

$$
\mu_{2 j}=\sum_{\Gamma}\left|\operatorname{Vx}(\Gamma) \cap\left\{1, \ldots, \mu_{2 j}\right\}\right| \leq \sum_{\Gamma}|\operatorname{Edge}(\Gamma)|=m_{j}
$$

Now let $X$ be the union of the component of $\operatorname{Gr}_{\pi}(s)$ which are contained in $\left\{1, \ldots, \mu_{2 j}\right\}$ and let $\Gamma$ be the component of $\operatorname{Gr}_{\pi}(s)$ which contains $\min (X)$. In particular $\operatorname{Vx}(\Gamma) \subseteq$ $\left\{1, \ldots, \mu_{2 j}\right\}$.

Consider the involution $\sigma_{\Gamma}:=\prod_{c \in \mathrm{Vx}(\Gamma)}(t(2 j-1, c), t(2 j, c))$. This transposes the entries between rows $2 j-1$ and $2 j$ in each column in $\operatorname{Vx}(\Gamma)$. Now it is clear that $\pi$ is in the row stabilizer of $\sigma_{\Gamma} s$. So $\left\{\sigma_{\Gamma} s\right\} \in \operatorname{supp}(\pi t) \cap \operatorname{supp}(t)$. Moreover, $\operatorname{Gr}_{\pi}(s)=\operatorname{Gr}_{\pi}\left(\sigma_{\Gamma} s\right)$ and $s=\sigma_{\Gamma}\left(\sigma_{\Gamma} s\right)$. It follows that the pair $T \neq \sigma_{\Gamma} T$ of tabloids makes zero contribution to $\left\langle\pi e_{t}, e_{t}\right\rangle$ modulo 2. But $T$ is an arbitrary $\pi$-fixed tabloid in $\operatorname{supp}(\pi t) \cap \operatorname{supp}(t)$. So $\left\langle\pi e_{t}, e_{t}\right\rangle$ is even, according to Lemma 8. This contradiction completes the proof.

\subsection{Proof of Theorem 2}

Suppose first that $P^{\mu}$ has quadratic type. Then by (ii) $\Longleftrightarrow(v)$ in Proposition 3 , $B(\hat{\pi} x, x\rangle \neq 0$, for some $x \in D_{A}^{\mu}$ and involution $\hat{\pi} \in 2 . \mathcal{A}_{n}$. Let $\pi$ be the image of $\hat{\pi}$ in $\mathcal{A}_{n}$. Then Remark 7 implies that there is a $\mu$-tableau $t$ such that $\left\langle\pi e_{t}, e_{t}\right\rangle$ is odd. Now $\pi$ is a $4 m$-involution, for some $m>0$, and Lemmas 9 and 10 imply that $\frac{n-|\mu|_{a}}{2} \leq 4 m \leq \frac{n-\ell_{o}(\mu)}{2}$. This proves the 'only if' part of the Theorem.

According to Lemma 4, the strongly real 2-regular conjugacy classes of 2. $\mathcal{A}_{n}$ are enumerated by $\lambda \in \mathcal{O}(n)$ such that there is a positive integer $m$ with $\frac{n-\ell(\lambda)}{2} \leq 4 m \leq$ $\frac{n-m_{o}(\lambda)}{2}$ (if $\lambda$ has distinct parts, $\frac{n-\ell(\lambda)}{2}=\frac{n-m_{o}(\lambda)}{2}$ and there are two 2-regular classes of 2. $\mathcal{A}_{n}$ labelled by $\lambda$, in all other cases there is a single 2 -regular class of $2 . \mathcal{A}_{n}$ labelled by $\lambda)$.

By Theorem 2.1 in [2] (or the main result in [9]) there is a bijection $\phi: \mathcal{O}(n) \rightarrow \mathcal{D}(n)$ such that $\ell(\lambda)=|\phi(\lambda)|_{a}$ and $m_{o}(\lambda)=\ell_{o}(\phi(\lambda))$, for all $\lambda \in \mathcal{O}(n)$. Then from the previous 
paragraph the number of strongly real 2-regular conjugacy classes of 2. $\mathcal{A}_{n}$ coincides with the number of irreducible $k\left(2 . \mathcal{A}_{n}\right)$-modules enumerated by $\mu \in \mathcal{D}(n)$ such that $\frac{n-|\mu|_{a}}{2} \leq 4 m \leq \frac{n-\ell_{o}(\mu)}{2}$ for some integer $m$. However, from earlier in the proof, these are the only $P^{\mu}$ which can be of quadratic type. We conclude from Proposition 1 that each of these $P^{\mu}$ is of quadratic type, and furthermore that no other $P^{\mu}$ is of quadratic type.

\subsection{Example with $2 . \mathcal{A}_{13}$}

The 18 distinct partitions of 13 give rise to 21 principal indecomposable $k\left(2 . \mathcal{A}_{13}\right)$ modules. The types are:

\begin{tabular}{|c|c|c|c|}
\hline$\mu$ & $\frac{n-||_{a}}{2}$ & $\frac{n-\ell_{o}(\mu)}{2}$ & type \\
\hline$(7,6)$ & 6 & 6 & 2 non-quadratic \\
$(8,5)$ & 5 & 6 & non-quadratic \\
$(6,5,2)$ & 5 & 6 & non-quadratic \\
$(6,4,2,1)$ & 5 & 6 & non-quadratic \\
$(5,4,3,1)$ & 5 & 5 & 2 not self-dual \\
$(7,5,1)$ & 5 & 5 & 2 not self-dual \\
$(9,4)$ & 4 & 6 & quadratic \\
$(7,4,2)$ & 4 & 6 & quadratic \\
$(6,4,3)$ & 4 & 6 & quadratic \\
$(8,4,1)$ & 4 & 6 & quadratic \\
$(7,3,2,1)$ & 4 & 5 & quadratic \\
$(10,3)$ & 3 & 6 & quadratic \\
$(8,3,2)$ & 3 & 6 & quadratic \\
$(9,3,1)$ & 3 & 5 & quadratic \\
$(11,2)$ & 2 & 6 & quadratic \\
$(10,2,1)$ & 2 & 6 & quadratic \\
$(12,1)$ & 1 & 6 & quadratic \\
$(13)$ & 0 & 6 & quadratic \\
\hline
\end{tabular}

Using (i) and (ii) in Lemma 5, we see that $D^{\mu} \downarrow_{\mathcal{A}_{13}}$ is a sum of two non-isomorphic irreducible $k\left(2 . \mathcal{A}_{13}\right)$-modules for $\mu=(7,6),(5,4,3,1)$ or $(7,5,1)$. For all other $\mu, D^{\mu} \downarrow_{\mathcal{A}_{13}}$ is irreducible. So there are $21=18+3$ projective indecomposable $k\left(2 . \mathcal{A}_{13}\right)$-modules.

By the last statement in Lemma 5 , the two irreducible $k\left(2 . \mathcal{A}_{13}\right)$-modules $D_{A}^{(5,4,3,1)}$ are duals of each other, as are the two irreducible $k\left(2 . \mathcal{A}_{13}\right)$-modules $D_{A}^{(7,5,1)}$. By the same result both irreducible $k\left(2 . \mathcal{A}_{13}\right)$-modules $D_{A}^{(7,6)}$ are self-dual. However $6 \equiv 2(\bmod 4)$. So neither principal indecomposable $k\left(2 . \mathcal{A}_{13}\right)$-module $P^{(7,6)}$ is of quadratic type.

Next if $\mu=(8,5),(6,5,2)$ or $(6,4,2,1)$ we have $\frac{n-|\mu|_{a}}{2}=5$ and $\frac{n-\ell_{o}(\mu)}{2}=6$. So the principal indecomposable $k\left(2 . \mathcal{A}_{13}\right)$-module $P^{\mu}$ is not of quadratic type for any 
of these $\mu$ 's. For each of the remaining partitions $\mu$, the principal indecomposable $k\left(2 . \mathcal{A}_{13}\right)$-module $P^{\mu}$ is of quadratic type, according to Theorem 2.

\section{References}

[1] D. Benson, Spin modules for symmetric groups, J. Lond. Math. Soc. (2) 38 (1988) $250-262$.

[2] W.Y.C. Chen, H.Y. Gao, K.Q. Ji, M.Y.X. Li, A unification of two refinements of Euler's partition theorem, Ramanujan J. 23 (1-3) (2010) 137-149.

[3] R. Gow, J. Murray, Quadratic principal indecomposable modules and strongly real elements of finite groups, arXiv:1803.03182 [math.RT], 8 Mar 2018.

[4] R. Gow, W. Willems, Quadratic geometries, projective modules and idempotents, J. Algebra 160 (1993) 257-272.

[5] B. Huppert, N. Blackburn, Finite Groups II, Grundlehren der math. Wissen., vol. 242, SpringerVerlag, 1982.

[6] G.D. James, The Representation Theory of the Symmetric Groups, Lecture Notes in Mathematics, vol. 628, Springer-Verlag, 2006.

[7] J.C. Murray, Symmetric bilinear forms and vertices in characteristic 2, J. Algebra 462 (2016) 338-374.

[8] J.C. Murray, The duals of the 2-modular irreducible modules of the alternating groups, SIGMA 14 (2018) 033, arXiv:1711.06009 [math.RT].

[9] J. Murray, A bijection for Euler's partition theorem in the spirit of Bressoud, arXiv:1803.11104 [math.CO], 29 March 2018.

[10] H. Nagao, Y. Tsushima, Representations of Finite Groups, Academic Press, 1988.

[11] J.R. Stembridge, Shifted tableaux and the projective representations of symmetric groups, Adv. Math. 74 (1989) 87-134.

[12] J. Thompson, Bilinear forms in characteristic $p$ and the Frobenius-Schur indicator, in: Group Theory, Beijing 1984, in: Lecture Notes in Math., vol. 1185, Springer-Verlag, 1984, pp. 221-230. 